\title{
SCHWANNOMA INTRALABERÍNTICO. DESCRIPCIÓN DE UN CASO
}

\section{Intralabyrinthine schwannoma. A case report}

\author{
José A. CÁMARA-ARNAZ ${ }^{1}$; José I. BENITO-OREJAS*; Margarita RODRÍGUEZ-VELASCO ${ }^{2}$; \\ Darío MORAIS-PÉREZ
}

Hospital Clínico Universitario. ${ }^{1}$ Servicio de Otorrinolaringología y Patología Cérvico-Facial. ${ }^{2}$ Servicio de Radiodiagnóstico. Valladolid. España.

*Correspondencia: jibenito@ono.com

Fecha de recepción: 26 de diciembre de 2015

Fecha de aceptación: 13 de febrero de 2016

Fecha de Publicación: 15 de febrero de 2016

Conflicto de intereses: Los autores declaran no tener conflictos de intereses Imágenes: Los autores declaran haber obtenido las imágenes con el permiso de los pacientes Política de derechos y autoarchivo: se permite el autoarchivo de la versión post-print (SHERPA/RoMEO) Licencia CC BY-NC-ND. Licencia Creative Commons Atribución-NoComercial-SinDerivar 4.0 Internacional @ C Universidad de Salamanca. Su comercialización está sujeta al permiso del editor

Introducción y objetivos: El schwannoma intralaberíntico es un tumor benigno muy infrecuente, de lento crecimiento extradural, localizado primariamente en el laberinto membranoso. A través de un caso clínico presentamos las características de su evolución, intentamos ofrecer una explicación fisiopatológica de la sintomatología y de los hallazgos audio-vestibulares y describimos finalmente, las diferentes opciones terapéuticas.

Descripción: Se trata de una mujer de 59 años, que comienza con síntomas inespecíficos de oído izquierdo a los que posteriormente se añade una pérdida auditiva. Al confirmar que se trata de una hipoacusia unilateral no justificada, solicitamos una RM que pone de manifiesto la presencia de un pequeño tumor de 2-3 mm intravestibular, al que revisaremos periódicamente. Discusión: El origen y la prevalencia del schwannoma intralaberíntico son desconocidos. Aunque la clínica se confunde con la de otros procesos otológicos más frecuentes, la realización de RM incrementaría el número de casos diagnosticados. En función del paciente, de su sintomatología, de la evolución y de las características tumorales, se decide el tipo de tratamiento Conclusión: El schwannoma intralaberíntico debe formar parte del diagnóstico diferencial en pacientes con síntomas audio-vestibulares unilaterales y solicitar una RM de alta resolución para obtener el diagnóstico definitivo. Presentando un pronóstico excelente, la observación es considerada como la mejor opción terapéutica.

PALABRAS CLAVE schwannoma; schwannoma vestibular; schwannoma intralaberíntico; hipoacusia; vértigo; resonancia magnética

SUMMARY

Introduction and objectives: The intralabyrinthine schwannoma is a rare benign tumor, slow growth extradural, located primarily in the membranous labyrinth. Through a clinical case we present the characteristics of its evolution, try to offer a physio-pathological explanation of symptoms and findings audio-vestibular and finally, describe the different therapeutic options. Description: It is a 59 year old woman, beginning with non-specific symptoms of the left ear and then concerned a hearing loss in that ear. To confirm that it is a unilateral hearing loss not justified, we request an MRI that shows the presence of a small tumor of 2-3 mm intravestibular, 
which we will review periodically. Discussion: The origin and the prevalence of intralabyrinthine schwannomas are unknown. Although the clinic is confused with other frequent otological processes, the implementation of MRI would increase the number of diagnosed cases. Depending on the patient, their symptoms, evolution and tumor characteristics, the type of treatment is decided. Conclusion: Intralabyrinthine schwannoma should be part of the differential diagnosis in patients with unilateral audio-vestibular symptoms and request a high resolution MRI to obtain the definitive diagnosis. Presenting an excellent prognosis, the observation is considered the best treatment option.

KEYWORDS

schwannoma; vestibular schwannoma; intralabyrinthine schwannoma; hearing loss; vertigo; magnetic resonance imaging.

\section{INTRODUCCIÓN}

El schwannoma vestibular (SV) (erróneamente denominado neurinoma del acústico, pues se origina de las células de Schwann y no de las fibras nerviosas; y asienta fundamentalmente en el nervio vestibular y no en el coclear) [1] es el tumor más frecuente del conducto auditivo interno (CAI) y del ángulo ponto-cerebeloso (APC). Excepcionalmente, de las ramas terminales del VIII par craneal, también puede surgir un schwannoma, que por su localización se denomina intralaberíntico (SIL), constituyendo un proceso clínico-patológico diferente al del SV [2].

Descritos a principios del s. XX como hallazgo autópsico, no es hasta el advenimiento de la $\mathrm{RM}$, en la década de los 90 , cuando se publica la primera serie de SIL diagnosticados preoperatoriamente [3]. Las revisiones más recientes [4] cifran en unos 340 los pacientes diagnosticados. Se trata de tumores benignos, de pequeño tamaño y lento crecimiento extradural, que afectan primariamente al vestíbulo, conductos semicirculares y/o cóclea, pudiendo excepcionalmente alcanzar el oído medio, o a través del CAI el APC. En 2004, Kennedy et al. [5], proponen una clasificación anatómica que basada en la localización y extensión tumoral, identifica 7 tipos: intravestibular, intracoclear, intravestíbulo-coclear, transmodiolar, transmacular, transótico y tímpano-laberíntico; distribución que fue aceptada por la comunidad neurootológica [6]. El origen intracoclear es el más frecuente $(51 \%)$ [2], aunque también pueden nacer, de las ramas terminales de los nervios vestibulares (intralaberíntico, 29\%).

Describimos el caso clínico de una paciente con un schwannoma intralaberíntico e intravestibular, puesto de manifiesto en el estudio radiológico. Relacionamos los hallazgos de la exploración audiovestibular con la localización tumoral.

\section{DESCRIPCIÓN}

Mujer de 59 años remitida en octubre de 2013 a nuestro Servicio, por presentar dolor esporádico y zumbido del oído izquierdo (OI). En sus antecedentes refería haber padecido de dicho oído en la infancia y desde hace unos 5 años presentar algún vértigo esporádico, sin síntomas auditivos. La exploración otoscópica mostraba un tímpano izquierdo cicatricial. El timpanograma era normal con presencia de reflejo estapedial ipsilateral (salvo a $4 \mathrm{kHz}$ en OI) y la audiometría tonal liminal dentro de los límites normales (Figura 1-1). Seis meses después, la paciente nos contó que no oía bien por el Ol. Se confirmó la presencia de una hipoacusia neurosensorial, con caída hacia los tonos agudos (Figura 1-2), que recuperó al cabo de un tiempo, de manera parcial (hipoacusia fluctuante). Las latencias de los potenciales evocados auditivos del tronco cerebral (PEATC), eran simétricas y normales (Figura 1). Se registraron otoemisiones acústicas (productos de distorsión), que desaparecieron en el OI a partir de $2 \mathrm{kHz}$. Clínicamente no se observó nistagmo espontáneo ni con fijación. La videooculografía era normal y en la prueba calórica bitérmica de Fitzgerald-Hallpike, había una arreflexia del oído izquierdo. Los potenciales evocados miogénicos vestibulares de origen cervical (cVEMPs), mostraban una amplitud y umbral simétricos entre ambos oídos (Figura 1). En la resonancia magnética (RM), aparecía una tumoración redondeada, de unos $2 \mathrm{~mm}$, entre el vestíbulo y los conductos semicirculares superior y externo, de baja señal en secuencia T2 y que captaba contraste (Figura 2). Se trataba de un posible schwannoma intravestibular y propusimos a la paciente una actitud expectante, con revisiones periódicas, que aceptó. 


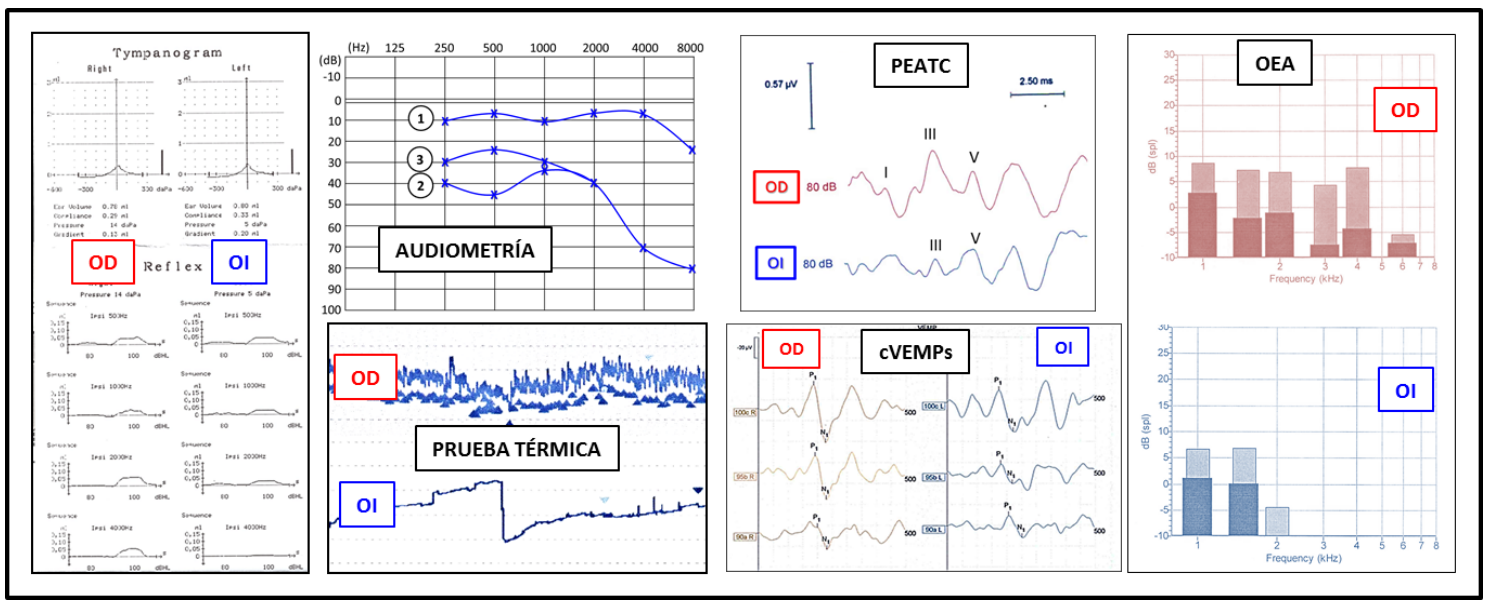

Figura 1. Hipoacusia fluctuante en tonos graves con descenso de los agudos (1: octubre 2013, 2: marzo 2014, 3: marzo 2015); timpanograma normal, con reflejos ipsi en todas las frecuencias menos en $4 \mathrm{kHz}$ del OI; PEATC con onda III-V simétrica; OEA (productos de distorsión) presentes en OD y en Ol sólo hasta $2 \mathrm{kHz}$; cVEMPs con amplitud simétrica y prueba térmica con arreflexia vestibular izquierda.

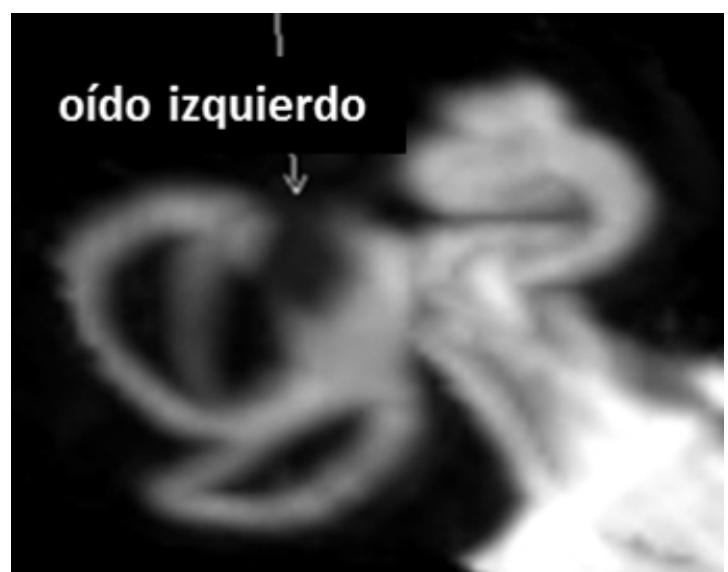

Figura 2. La reconstrucción tridimensional de la RM permite visualizar la pequeña lesión del vestíbulo izquierdo (schwannoma intravestibular).

\section{DISCUSIÓN}

En todos los nervios craneales y espinales, con excepción del cocleovestibular (VIII par), la neuroglía se extiende tan solo una fracción de milímetro más allá de su origen en el tronco del encéfalo o la médula espinal. En el VIII par, la glía abarca una longitud media de $9,75 \mathrm{~mm}$ a lo largo de su trayecto [1]. La intersección del tejido glial a las células de Schwann se denomina "zona de unión glial-Schwann» o "zona de Obersteiner-Redlich», en memoria de quienes la describieron [1]. Esta área transicional se suele localizar a nivel del poro acústico interno, considerándose el lugar de nacimiento de los schwannomas vestibulares (SV), aunque sin evidencias científicas, pues cuando se opera un tumor, su crecimiento hace imposible reconocer el origen exacto. Pero además, el SV representa el $90 \%$ de los schwannomas originados en todos los pares craneales, originándose principalmente en la porción vestibular del nervio (con una razón en relación al coclear de 3,2 a 1) [1].

Sin embargo y de forma peculiar, los schwannomas del VIII par también pueden emerger de las ramas terminales del nervio, denominándose en estos casos schwannomas intralaberínticos (SIL). En los axones del nervio coclear, la capa de mielina se extiende hasta cerca del ganglio espiral. Se postula que la mayoría de los SIL $(51 \%)$ se originarían en el modiolo de la vuelta basal de la cóclea (intracocleares) [2] creciendo hacia la escala timpánica, probablemente por su estrecha relación con las ramas nerviosas [7]. Desde la escala timpánica, estos tumores podrían extenderse a la vestibular y desde la rampa basal a la altura de la ventana redonda, llegar al vestíbulo por los espacios perilinfáticos del sáculo. La invasión contraria, desde el vestíbulo a la cóclea, seguiría la misma ruta. De forma excepcional, atravesando la ventana redonda alcanzarían el oído medio o a través de la espira cribiforme y del CAI, llegarían al APC [7]. Un $29 \%$ de SIL son de origen intravestibular y nacen de las ramas terminales del nervio vestibular pudiendo desarrollarse en el nervio sacular, utricular, o en los nervios ampulares lateral, superior o posterior [2].

La etiología de estos tumores intralaberínticos es desconocida, con posible influencia genética y su prevalencia es muy escasa [8], aunque se supone que la realización de una RM de alta resolución ante síntomas audio-vestibulares unilaterales, permitiría descubrir más casos [5]. 
La edad media de diagnóstico del SIL es de 49 años (rango 14 a 89 años), sin predilección de sexo [8]. Dada su rareza, la clínica inespecífica y las dificultades de detección radiológica, el promedio de tiempo transcurrido hasta el diagnóstico es de unos 5-7 años [2, 8], lo que favorece la extensión tumoral [2].

Los síntomas predominantes son, la hipoacusia neurosensorial unilateral $(99 \%)$ (con sordera profunda en el $75 \%$ de casos), acúfenos $(65 \%)$ y síntomas vestibulares, mimetizando la de otros procesos neurootológicos más frecuentes [2, 8].

La configuración audiométrica de la hipoacusia es variable y su evolución puede ser progresiva $(61 \%)$, brusca (32\%) o fluctuante (7\%) [8]. El que en un $30 \%$ no se afecte la cóclea y aparezca hipoacusia, como en el caso presentado, significa que deben existir otros mecanismos fisiopatológicos, compresivos, vasculares (que podrían facilitar la producción de un hidrops endolinfático), neuronales o metabólicos (por una supuesta liberación tumoral de toxinas o iones potasio) [4], responsables. Incluso pudiera aparecer una hipoacusia mixta, denominada, pérdida conductiva del oído interno [5], secundaria a la interferencia del tumor con los fluidos cocleares o con el estribo. Nos resulta atractiva la hipótesis que pretende explicar la evolución de la hipoacusia en los SIL, dependiendo de la localización. Así, en tumores intracocleares, deberíamos esperar la presencia de una hipoacusia neurosensorial coclear y progresiva, secundaria a la compresión directa del órgano de Corti, pudiendo manifestarse un patrón retrococlear o una hipoacusia brusca en casos de compresión del nervio coclear o de su aporte vascular [9]. De la misma manera, la hipoacusia que pudiera aparecer en la localización intravestibular, sería con más frecuencia fluctuante y secundaria a un hidrops endolinfático causado por la compresión tumoral del «ductus reuniens» o del sáculo [9].

Los síntomas vestibulares son variables: vértigo episódico (59\%), mareo inducido por el movimiento y desequilibrio [8]. Conocer el origen del vértigo es complicado, porque incluso en un $36 \%$ de pacientes con este síntoma, la localización del schwannoma es exclusivamente coclear [10]; sin embargo el $100 \%$ de los pacientes con tumores del sistema vestibular, muestran hallazgos anómalos en la exploración física [2], como se corrobora en nuestro caso, con la arreflexia vestibular. En resumen, la clínica no deriva directamente de la localización y el tamaño tumoral, porque la compresión no sólo produce un efecto local, sino que puede también generar hidrops endolinfático, aplastamiento nervioso, afectación vascular e incluso cambios metabólicos locales, que podrían explicar el resto de manifestaciones [4]. Al no existir síntomas o signos patognomónicos del proceso, el diagnóstico definitivo es por $\mathrm{RM}$ de alta resolución (1,5 T o $3 \mathrm{~T}$ ). La primera serie de casos evaluados por RM antes de la cirugía fue publicada en 1994 [3]. Para su realización, se utilizan secuencias 3 DT2 (FIESTA, DRIVE) donde la lesión se delimita mejor como defecto de repleción, con baja señal en el seno del líquido laberíntico y 3 DT1 con gadolinio para comprobar su marcado realce, porque ambos hallazgos son necesarios para el diagnóstico. Los avances en resolución permiten detectar, como en el caso presentado, lesiones de $2-3 \mathrm{~mm}$ y si no se aplica este tipo de secuencias, un protocolo rutinario de RM podría dejar pasar la presencia de este tumor [6-7, 11-12].

Aunque las exploraciones audiológicas y vestibulares no son específicas de este proceso [3], en nuestra paciente, la arreflexia térmica en la prueba bitérmica de Fitzgerald-Hallpike sería consecuencia de la afectación tumoral del utrículo y los conductos semicirculares superior y externo. Por otra parte, al no alcanzar el tumor ni el conducto semicircular posterior ni el sáculo, se respeta la funcionalidad del nervio vestibular inferior, lo que nos permite obtener unos cVEMPs simétricos. Finalmente, la hipoacusia fluctuante podría ser consecuencia de un daño coclear indirecto, dada la ausencia parcial de otoemisiones acústicas, con PEATC normales [13] (Figura 1). Siguiendo la hipótesis previa [9], si se alteraran posteriormente los PEATC, podríamos sospechar que el tumor ha llegado al CAI, manifestándose la alteración retrococlear como una consecuencia de la compresión nerviosa.

El diagnóstico diferencial en función de la sintomatología se puede hacer con la enfermedad de Ménière, la migraña vestibular, la laberintitis y neuritis vestibular, la hipoacusia autoinmune, otosífilis y schwannoma vestibular [8]. La primera recopilación de 23 casos con este tumor se publicó en 1988 [14], de los que el $44 \%$ habían sido incorrectamente diagnosticados de enfermedad de Ménière. A nivel de RM las imágenes se deben diferenciar de la laberintitis, lipoma, quiste dermoide, granuloma y hemorragia intralaberíntica $[2,5,8,12]$.

El tratamiento depende de la sintomatología, tamaño tumoral, localización, edad y preferencias del paciente [4, 8]. La actitud habitual, es esperar y valorar con RM el crecimiento [2]. El resto de estrategias activas incluirían la resec- 
ción microquirúrgica, la radiocirugía estereotáxica y la laberintectomía química [2]. Las indicaciones quirúrgicas del SIL son, en principio, el vértigo intratable, el crecimiento tumoral dentro del CAI/APC o del oído medio, la pérdida de audición útil (se considera útil cuando el umbral tonal medio es mejor de $50 \mathrm{~dB} \mathrm{HL}$ o el porcentaje máximo de discriminación verbal es superior al 50\%) [15] y la duda diagnóstica $[2-5,8,9,16]$. Debemos tener en cuenta que cualquier acto quirúrgico para extirpar estos tumores sacrificará la audición [2-5, 8-9].

En la serie de Van Abel et al. (2013) [2] un 53\% de pacientes fueron observados, (un 52\% de los que fueron seguidos con RM, presentó crecimiento tumoral, pero sólo el 3\% necesitó tratamiento quirúrgico, por vértigo intratable o crecimiento tumoral, lo que apoya mantener una actitud expectante) [10]. En estos casos la conducta general consistiría en repetir la $\mathrm{RM}$ al año y si no ha habido crecimiento, realizarla cada 2 años. En el supuesto de vértigo severo en un paciente que no se quisiera operar o tuviera alguna contraindicación quirúrgica o mantuviera una audición útil, se puede usar, como tratamiento sintomático, la inyección intratimpánica de gentamicina, a sabiendas de que no se influye sobre el crecimiento tumoral $[12,15]$. El vértigo cede siempre con el tratamiento quirúrgico (se supone que la resección tumoral suprime la señal periférica alterada); pero también mejora en un $22 \%$ de pacientes que sólo son observados, quizá como consecuencia de una adaptación del sistema vestibular a lo largo del tiempo [4]. Siempre podremos utilizar el tratamiento médico y la rehabilitación hasta una posible cirugía.

Si se opta por el tratamiento quirúrgico, el tipo de cirugía dependerá de la localización tumoral $[2,4,5,8,17]$. Si el tumor se encuentra exclusivamente en el sistema vestibular se puede realizar un abordaje transmastoideo y una laberintectomía total. Si la ubicación es coclear, el acceso será transótico, requiriendo el cierre posterior del oído medio, para evitar la fístula de LCR desde el CAI a través del modiolo. Pero la indicación quirúrgica depende del nivel de audición y de la edad. No estaría indicado en casos que mantengan una audición útil y en los pacientes de edad avanzada, hay que tener en cuenta la mayor morbilidad y el hecho de que el crecimiento tumoral es más lento [4]. Como excepción, se aconsejaría la cirugía precoz si el diagnóstico se realiza en una persona joven ( $\leq 50$ años), pues aunque el crecimiento fuera de $1 \mathrm{~mm}$ cada 2-3 años, después de 30 años precisaría cirugía y sería un paciente anciano con mayores riesgos [8].
A diferencia del schwannoma vestibular (SV), en los intralaberínticos (SIL) no se recomienda la radiocirugía [15]. La preservación auditiva probablemente no sea posible en los SIL dadas las altas dosis de radiación que recibe la cóclea, además de que el lento crecimiento de estos tumores dificulta evaluar su efectividad. Asimismo, la cirugía genera menor riesgo iatrogénico de lesión en el nervio facial, que la tasa de parálisis postradiación. Sólo sería considerada cuando los síntomas impusieran la necesidad de cirugía y no se pudiera operar [15].

Hasta el momento, ninguno de los pacientes intervenidos ha tenido recurrencia y las complicaciones post-quirúrgicas son raras, con una afectación del nervio facial del 4\% [8].

Finalmente, en cuanto a la rehabilitación auditiva, la posibilidad sería un implante coclear (IC). Si el tumor es intralaberíntico, se podría colocar en la misma intervención, pero si es intracoclear, es improbable que el IC funcione [18]. En este caso, otra posibilidad para evitar el efecto sombra de la cabeza, sería colocar un implante osteointegrado [19] o usar un «sistema cross».

\section{CONCLUSIONES}

Los schwannomas intralaberínticos son tumores muy infrecuentes, cuya sintomatología se confunde con la de otros procesos neurootológicos más habituales. El diagnóstico definitivo sólo es posible a través de una RM de alta resolución. La observación es el planteamiento habitual en caso de audición útil y si se indica la cirugía, la extirpación tumoral se realizará a través de un abordaje translaberíntico o transótico, según la localización. El pronóstico de estos tumores es excelente.

\section{BIBLIOGRAFÍA}

1. Roosli C, Linthicum FH Jr, Cureoglu S, Merchant SN. What is the site of origin of cochleovestibular schwannomas?. Audiol Neurootol. 2012;17:121-5.

2. Van Abel KM, Carlson ML, Link MJ, Neff BA, Beatty CW, Lohse CM, et al. Primary inner ear schwannomas: a case series and systematic review of the literature. Laryngoscope. 2013;123:195766.

3. Doyle KJ, Brackmann DE. Intralabyrinthine schwannomas. Otolaryngol Head Neck Surg. 1994;110:517-23. 
4. Gosselin É, Maniakas A, Saliba I. Metaanalysis on the clinical outcomes in patients with intralabyrinthine schwannomas: conservative management vs. microsurgery. Eur Arch Otorhinolaryngol. 2015. Feb 12. [Epub ahead of print].

5. Kennedy RJ, Shelton C, Salzman KL, Davidson HC, Harnsberger HR. Intralabyrinthine schwannomas: diagnosis, management, and a new classification system. Otol Neurotol. 2004;25:1607.

6. Salzman KL, Childs AM, Davidson HC, Kennedy RJ, Shelton C, Harnsberger HR. Intralabyrinthine schwannomas: imaging diagnosis and classification. AJNR Am J Neuroradiol. 2012;33:104-9.

7. Tieleman A, Casselman JW, Somers T, Delanote J, Kuhweide R, Ghekiere J, et al. Imaging of intralabyrinthine schwannomas: a retrospective study of 52 cases with emphasis on lesion growth. AJNR Am J Neuroradiol. 2008;29:898905.

8. Frisch CD, Eckel LJ, Lane JI, Neff BA. Intralabyrinthine Schwannomas. Otolaryngol Clin North Am. 2015;48:423-41.

9. Lee JJ, Lim HJ, Park HY, Choung YH. Recovery of hearing after surgical removal of intralabyrinthine schwannoma. Laryngoscope. 2015;125:1968-71.

10. Fitzgerald DC, Grundfast KM, Hecht DA, Mark AS. Intralabyrinthine schwannomas. Am J Otol. 1999;20:381-5.

11. Dubrulle F, Kohler R, Vincent C, Puech $P$, Ernst $O$. Differential diagnosis and prognosis of T1-weighted post-gadolinium intralabyrinthine hyperintensities. Eur Radiol. 2010;20:2628-36.
12. Alemi AS, German MA, Hasso AN, Djalilian HR. Radiology quiz case 3. Intralabyrinthine schwannomas (ILS). Arch Otolaryngol Head Neck Surg. 2011;137:825, 827-8.

13. Nishimura T, Hosoi H. Progressive hearing loss in intracochlear schwannoma. Eur Arch Otorhinolaryngol. 2008;265:489-92.

14. Sataloff RT, Roberts BR, Feldman M. Intralabyrinthine schwannoma. Am J Otol. 1988;9:323-6.

15. Neff BA, Willcox Jr TO, Sataloff RT. Intralabyrinthine schwannomas. Otol Neurotol. 2003;2:299-307.

16. Maseda E, Ablanedo A, Blanco R, Díaz C, Martín A. Schwannoma intralaberíntico: a propósito de dos casos. Acta Otorrinolaringol Esp. 2007;58:70-2.

17. Bouchetemblé $P$, Heathcote $K$, Tollard E, Choussy O, Dehesdin D, Marie JP. Intralabyrinthine schwannomas: a case series with discussion of the diagnosis and management. Otol Neurotol. 2013;34:944-51.

18. Schutt CA, Kveton JF. Cochlear implantation after resection of an intralabyrinthine schwannoma. Am J Otolaryngol. 2014;35:257-60.

19. Jia H, Marzin A, Dubreuil C, Tringali S. Intralabyrinthine schwannomas: symptoms and managements. Auris Nasus Larynx. 2008;35:131-6. 